\title{
Communication Mode Selection and Pricing Mechanism for Relaying Based Device-to-Device Communications
}

\author{
Fan Jiang ${ }^{1}$, Benchao Wang ${ }^{2}$ and Changyin Sun ${ }^{1}$ \\ ${ }^{1}$ School Of Communications And Information Engineering, \\ Xian University Of Posts and Telecommunications, Xi'an, 710121, P. R. China \\ ${ }^{2}$ Shaanxi Radio Monitoring Station, \\ State Radio Regulatory of China, Xi'an 710200, P. R. China \\ Ifjiangwbc@gmail.com,wbc515@163.com
}

\begin{abstract}
In this paper, the communication mode selection issue as well as pricing mechanism in relaying enhanced device-to-device (D2D) communications is discussed. By considering the user density within the cell, the problem of "when D2D relaying mode should be selected?" is explored. Furthermore, based on game theoretical approach, the optimal pricing strategy is proposed so as to encourage D2D relaying behavior among D2D peers. Considering user's demands and D2D devices' constraint ability, the proposed mechanism is based on the user's payoff model. Simulation results demonstrate that under game theoretical model, the proposed scheme can achieve the maximal utility for D2D devices when the reasonable price is adopted, hence better user experience can be obtained.
\end{abstract}

Keywords: Device-To-Device, Communication Mode Selection, Pricing Strategy, NonCooperative Game, Cooperative Game

\section{Introduction}

With the rapid growth of advanced wireless communication technologies, wireless networks have been widely accepted as an inexpensive solution to provide last mile access to the Internet. At the same time, user demands for mobile broadband are undergoing an unprecedented rise[1-2]. In China alone, the fast growth and widely use of instant messaging applications such as Wechat and Weibo for multimedia file sharing and online video browsing are already challenging the limits of current cellular systems. The ever growing demand for higher data rates and capacity requires new technology for the next generation $(5 \mathrm{G})$ cellular systems.

To alleviate the huge infrastructure investment with the exponential growth of mobile service and improve local service flexibility, device-to-device (D2D) communications have been proposed as one of the key techniques in the Third Generation Partnership Project (3GPP) Long Term Evolution Advanced (LTE-Advanced). The principle of D2D communications is shown in Figure1. From the figure, user equipment (UE) nearby can communicate either directly or access the Base Station (BS) through D2D relaying. Due to the physical proximity, this new communication paradigm provides significant performance gains in terms of spectral efficiency, energy efficiency and system capacity. However, unlike the existing short-range wireless transmission techniques (e.g., Wi-Fi, Bluetooth), D2D communications usually utilize the licensed spectrum bands [3], thus can guarantee uniform service provision. Furthermore, D2D communications can provide offload function and avoid congestion in the existing cellular network.

Since the potential benefits of D2D communications for both operators and users still need to be explored, extensive studies have been carried out in the literature. In [4], the basic concept of D2D communications is introduced, and the technical challenges and 
potential applications facing with D2D communications have been addressed. In [5], the tight integration of D2D communications with LTE-Advanced network has been proposed, the authors also discussed the D2D communications session setup and management procedures in the LTE-Advanced networks. In [6], the challenges and four possible D2D communication scenarios and the future research directions of D2D communications in $5 \mathrm{G}$ cellular networks have been introduced. The advantages and disadvantages of both D2D direct communications and D2D relaying have been analyzed. Moreover, the article also proposes some pricing schemes for device relaying in D2D communications scenario.

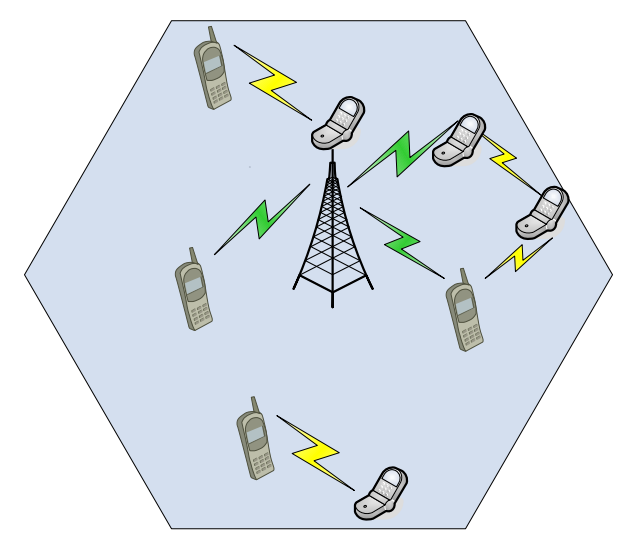

Figure 1. D2D Communications Scenario

When related to the scenario of D2D relaying communications [7], although some of current researches have already discussed the procedure of how devices can discover each other to form the D2D relaying peer[8], one critical issue often been omitted is that under which condition should UEs switch to D2D communication mode. In order to carry out D2D relaying, besides link conditions and user's requirement, an indispensible requirement is whether there exists certain device to be selected. Furthermore, once the D2D relaying mode has been chosen, the pricing mechanism is also a great challenge. Since device use its own resource for D2D relaying, it is reasonable and practicable that it should charge for its relaying service. However, a dilemma lies in how should a device bids a reasonable service price that not only satisfying UE's requirement, but also maximize its own utility. Moreover, what is the optimal policy of BS in order to encourage D2D relaying behavior?

To this ends, and by taking the user's density into account, this paper firstly answers the question of "when should D2D relaying mode been chosen?" Moreover, based on non-cooperative game theory as well as cooperative game theory, we propose the optimal pricing mechanism for D2D communication peers to answer the question of "How should D2D relaying behavior been encouraged?" The proposed mechanism is based on the user's payoff model which considers both user device's demands and constraint ability.

We structure the article as follows. Considering the impact of user density, Section 2 analyzes the D2D communication mode selection criteria. Section 3 first proposes the user's payoff model. Furthermore, based on non-cooperative game theory as well as cooperative game theory, we arrive to the optimal pricing mechanism. The numerical results are provided in Section 4. Finally, Section 5 concludes the paper. To clarify user's roles, we denote the user who initiates D2D communication as "UE", while users participate in D2D relaying as "devices". 


\section{The Criteria for D2D Relaying Mode Selection}

\subsection{Analytical Model}

According to the conventional BS relaying scheme, UE directly transmits its information to BS in the uplink. However, suppose at a certain time $t$, the direct communication link between UE and BS is not good enough for the BS to correctly decode the message, and there is no other low power nodes (e.g., fixed relay node, femto cell) deployed to help relaying the message. In order to find another device for message relaying, UE will consider switch to D2D communication mode. Hence, we assume the transmission scenario that a UE transmit to BS through D2D relaying, which is an important paradigm in D2D transmission type [8]. Before the peer-to-peer D2D device discovery process, an essential problem needed to be solved is whether there exists certain device for D2D communications. In other words, we need to figure out when UE should adopt D2D relaying mode. Therefore, we will focus on this issue and propose the criteria for D2D relaying mode transition.

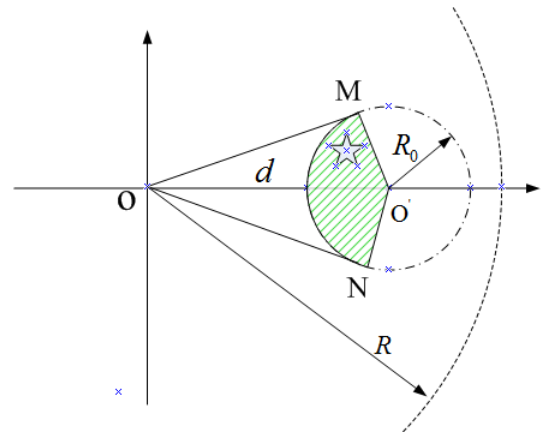

Figure 2. Analytical Model of D2D Relaying Mode

The analytical model of D2D relaying mode selection is described in Figure 2. Assume that a circle centered at position $O$ and with a radius $R$ denotes a cell. Within this cell, the BS is located in the center. Suppose at a certain time $t$, a certain $\mathrm{UE}_{\mathrm{i}}$ located at position $o$ in Figure 2 and its distance to the BS is $d$. Furthermore, the transmission ranges of $\mathrm{UE}_{\mathrm{i}}$ can be represented by a circle with a radius $R_{0}$ which is centered at $o^{\prime}$. The shaded region within the $\mathrm{UE}_{\mathrm{i}}{ }^{\prime}$ 's coverage circle represents $\mathrm{UE}_{\mathrm{i}}{ }^{\prime}$ 's effective D2D communication area (EDCA), where $\angle \mathrm{OMO}^{\prime}=\angle \mathrm{ONO}^{\prime}=\pi / 2$. Only if there exists at least another device (referred to as the "star" shown in Figure 2) within its EDCA, $\mathrm{UE}_{\mathrm{i}}$ is eligible to initiating a D2D relaying mode transition.

\subsection{D2D Communication Transition Threshold}

Suppose that all the UEs arrive to the cell independently, and UEs are evenly distributed within the cell. At a certain time $t$, the user density within the cell is $\eta$ per unit area. The average number of UEs within the cell then can be calculated as $\pi R^{2} \eta$. According to Figure 2, let $D_{E}(d)$ represents $\mathrm{UE}_{\mathrm{i}}$ 's EDCA, the probability that $\mathrm{UE}_{\mathrm{i}}$ can find a D2D device within its EDCA is a function of a binomial distribution with parameters $\left(\pi R^{2} \eta, D_{E}(d) / \pi R^{2}\right)$. In these parameters, $D_{E}(d) / \pi R^{2}$ denotes the probability that an arbitrary device is located within the $\mathrm{UE}_{\mathrm{i}}$ 's EDCA. According to [9], the value of $D_{E}(d)$ is given by: 


$$
D_{E}(d)=\pi R_{0}^{2} \times \frac{\cos ^{-1}\left(R_{0} / d\right)}{\pi}=R_{0}^{2} \times \cos ^{-1}\left(R_{0} / d\right)
$$

As a result, the probability that $\mathrm{UE}_{\mathrm{i}}$ can find $m$ number of $\mathrm{D} 2 \mathrm{D}$ devices within its EDCA is written as:

$$
\left.\psi(m)=\left(\begin{array}{l}
\left.\pi R^{2} \eta\right) \\
m
\end{array}\right)\left|\frac{R_{0}^{2} \times \cos ^{-1}\left(R_{0} / d\right)}{\pi R^{2}}\right|\left|1-\frac{R_{0}^{2} \times \cos ^{-1}\left(R_{0} / d\right)}{\pi R^{2}}\right|\right)^{\pi R^{2} \eta-m}
$$

As mentioned above, a certain time $t$, if $\mathrm{UE}_{\mathrm{i}}$ successfully finds another device in its EDCA, then $\mathrm{UE}_{\mathrm{i}}$ can switch to D2D mode and begin a peer-to-peer discovery. This is given by the following expression:

$$
1-\psi(0) \geq \psi(0)
$$

Substituting (2) into (3), we have:

$$
\left\{1-\frac{R_{0}^{2} \times \cos ^{-1}\left(R_{0} / d\right)}{\pi R^{2}} \mid \geq \frac{1}{2}\right.
$$

In this formula, the minimal value of $\eta$ that satisfies (4) is set as the D2D relaying mode transition threshold $\eta_{0}$. Whenever a UE want to start D2D communications, it should first check the current user density. If $\eta \geq \eta_{0}$, for example, in the dense populated public place like shopping mall or theater, it might be easily to find another device to carry out D2D communications. While on the other hand, when $\eta<\eta_{0}$, it is recommended that D2D communication mode should not be selected since UE might not possibly found a suitable device within its EDCA.

\section{The Pricing Mechanism for D2D Relaying}

After D2D mode has been selected, another dilemma lies in how to encourage devices to participate in D2D relaying communications. However, unlike fixed relaying issues, devices participating in D2D relaying have only limited resources such as battery, data storage, and bandwidth. Therefore D2D relaying should consider both different candidate devices' ability and UE's preference as well.

Moreover, as mentioned above, devices that act as relays for other users use their own resources. They should get a decent payback from UEs who initiate the D2D relaying. Otherwise, if the charged/payoff for D2D services is not lucrative enough, these devices may turn down the D2D relaying request, and UEs who call for D2D relaying may end up with traditional BS relaying mode, which has lower speed and less efficiency. Therefore, after D2D mode selected, effective pricing models should also be designed to encourage devices to participate in this type of communication.

\subsection{Optimal Pricing Method Based On Non-Cooperative Game}

In this section, with regard to user's demands and abilities, we will consider the pricing strategy of devices participating in D2D communications. Based on both cooperative and non-cooperative game theory, we make the following assumptions without loss of generality: (i) devices have limited bandwidth resource to provide relaying services; (ii) devices compete with each other by setting relaying service prices for more revenue; (iii) 
the relay service provided by different devices is replaceable; (iv) there exists no coalition between different devices.

\subsubsection{Playoff Model}

Under these assumptions, the relation of devices in the cell can be modeled by noncooperative Bertrand game model [10]. The players of the game model are chosen the suitable D2D relaying device. Here, we assume at time $t$, the total number of devices that want to and can be selected to carry out D2D relaying is $K$. Then, the corresponding strategies of a certain device $i$ is proposing charging price $P_{i}$. While for certain UE, the strategy is choosing the best device peer among $K$ devices who can satisfy UE's Quality of Service(QoS). Consequently, the payoff model of the device $i$ who are competing with other devices for providing D2D relaying can be defined as

$$
R_{i}(\boldsymbol{P})=\left(P_{i} D_{i} Q_{i}-c_{i} Q_{i} D_{i}+r_{i}\right)
$$

Where $\boldsymbol{P}=\left\{P_{1}, \cdots P_{i}, \cdots P_{K}\right\}$ denote the service price offered by $K$ devices. $D_{i}$ denotes desired amount of QoS requirements of the UE which initiate D2D relaying. Since each device has limited ability, it is possible that device $i$ might satisfy only partial of UE's D2D relaying requirement. Therefore, $Q_{i}$ is introduced to represent the efficiency of $D_{i}$, that is, how much portion of UE's requirement would be fulfilled. Hence, $D_{i} Q_{i}$ is interpreted as the percentage or portion of UE's desired amount of requirement that can be met through D2D relaying by device $i$. Denote $c_{i}$ as the cost factor of device $i$ to serve as the D2D relaying peer. Moreover, in order to encourage D2D relaying behavior, it is assumed that device $i$ will obtain the revenue $r_{i}$ from BS for providing D2D relaying service.

With $K$ devices participate in D2D relaying, the total revenue of the network then can be expressed as

$$
R_{\text {Total }}(\boldsymbol{P})=\sum_{i=1}^{K} R_{i}
$$

\subsubsection{Utility Functions}

Under the assumption that the D2D relaying service provided by different devices in cellular network is replaceable and differentiated, the D2D relaying service in the cellular network can be modeled as an oligopoly market producing differentiated but substitutability goods. It is obvious that the total utility of all devices (competitors) should be a function containing both UE's demand $D_{i}$ and the corresponding charging price $P_{i}$. More specifically, it should be a second-order differentiable and strictly concave function of user demand. According to [11], the quadratic utility function originated from economics theory meets the requirements of utility function of D2D relaying, and can be applied to model the utility of devices in this paper.

Let $U(\mathbf{D})$ represents the total utility function of devices within the cellular network, with above considerations, $U(\mathbf{D})$ should be set up as:

$$
U(\mathbf{D})=\sum_{i=1}^{K} D_{i} Q_{i}-\frac{1}{2}\left(\sum_{i=1}^{K}\left(D_{i} Q_{i}\right)^{2}+2 \rho \sum_{i \neq j} D_{i} Q_{i} D_{j} Q_{j}\right)-\sum_{i=1}^{K} P_{i} D_{i} Q_{i}
$$

Where $\mathbf{D}=\left\{D_{1}, \cdots D_{i}, \cdots D_{K}\right\}$ denote the demand vector of all the $K$ devices. Parameter $\rho$ denotes the competition factor among devices, where $0 \leq \rho \leq 1$. When $\rho=0$, this means there is only one device that could meets the UE's minimum requirement, 
whereas $\rho=1$ indicates that the qualities of all device's relaying service are identical. Hence, $0 \leq \rho \leq 1$ represents the competitive degree of substitutable relaying service among devices.

In order to obtain the maximal utility of all devices, and according to given charging price $P_{i}$, the optimal value of $D_{i}$ should be resolved. The optimal $D_{i}$ leading to maximal $U(\mathbf{D})$ can be obtained by differentiating $U(\mathbf{D})$ with respect to $D_{i}$ and set the ratio to zero.

$$
\frac{\partial U(D)}{\partial D_{i}}=1-D_{i} Q_{i}-\rho \sum_{i \neq j} D_{j} Q_{j}-P_{j}=0
$$

By jointly solve function (8) for each $D_{i}, i=1,2 \ldots, K$, the optimal demand function can be obtained as :

$$
D_{i}\left(P_{i}\right)=\frac{\left(1-P_{i}\right)[\rho(K-2)+1]-\rho \sum_{i \neq j}\left(1-P_{i}\right)}{Q_{i}(1-\rho)[\rho(K-1)+1]}
$$

\subsubsection{Nash Equilibrium of Non-Cooperative Game}

Given the optimal demand function shown in (9), it can be observed that each UE's optimal demand is closely related to the price $P_{i}$ charged by D2D relaying device $i$. Consequently, with the variation of charging price $P_{i}$, the corresponding optimal demand of each UE also varies. For a $K$-player non-cooperative game, the Nash equilibrium point of the non-cooperative game is the strategy profile that no player can increase his or her payoff by choosing another scheme while other players' action remains unaffected [12]. Thus, the Nash equilibrium price can be obtained through the best response function of each player in the non-cooperative game.

According to the revenue function in (5) and the demand function in (9), by considering the optimal demand, each device's best strategy is to get larger revenue by meeting UE's requirement through D2D relaying service. The revenue issue of each device can be then expressed as a function of charging price. By substituting equation (9) in to equation (5), we have

$$
\left.R_{i}\left(P_{i}\right)=r_{i}+\left(P_{i}-C_{i} Q_{i}\right) \backslash \frac{\left\lceil\left(1-P_{i}\right)[\rho(K-2)+1]-\rho \sum_{i \neq j}\left(1-P_{j}\right)\right.}{Q_{i}(1-\rho)[\rho(K-1)+1]}\right\rfloor
$$

In order to obtain the optimal charging price $P_{i}$, by differentiating $R_{i}\left(P_{i}\right)$ in (10) with respect to $P_{i}$, it follows that

$$
\frac{\partial R_{i}\left(P_{i}\right)}{\partial P_{i}}=0
$$

Consequently, the optimal charging price represented by $P_{i}^{*}$ can be obtained as

$$
P_{i}^{*}=\frac{1}{2}\left(1+C_{i} Q_{i}\right)-\frac{\rho \sum_{i \neq j}\left(1-P_{i}\right)}{2 \rho(K-2)+2}
$$


By jointly solving equation (12), the best charging price (BCP) $P_{i}^{*}$ of the each D2D relaying device can be obtained similarly. Consequently, the Nash equilibrium price of the $K$ competed D2D relaying devices can be obtained.

\subsection{Optimal Pricing Method Based On Cooperative Game}

\subsubsection{Cooperative Game Model}

In aforementioned model, we assume that devices are competing with each other for providing D2D relaying, so as to attract more users to increase their revenue, especially when the service quality and price offered by different devices are comparable and these devices belong to different Service Providers (SP). However, since severe competition among devices may result in lower service price and shrink total profits, which may be not acceptable for the devices performing D2D relaying. In the competitive wireless service environment, all or part of devices may turn to coalition instead of competition. Moreover, there is also another possibility that some of the devices belong to certain SP, which has the same profit goal. In order to characterize the strategies and payoffs for such situation, a cooperative game model can be applied [12]. In this model, in order to gain more profits, several different devices cooperate with each others in an oligopoly market through selling the same quality of D2D relaying service to user at the coalition price; therefore the strategies chosen by each device is the service price and is set to be the same price.

Similarly to the definitions in (5) and (6), under cooperative game model, the payoff $R_{i}^{C}(P)$ and the total revenue $R_{i}^{\text {Total }}(P)$ are defined, respectively, as follows:

$$
\begin{aligned}
R_{i}{ }^{C}(\boldsymbol{P}) & =\left(P_{i}{ }^{C} D_{i}{ }^{C} Q_{i}-c_{i} Q_{i} D_{i}{ }^{C}+r_{i}\right) \\
R_{\text {Total }}{ }^{C}(\boldsymbol{P}) & =\sum_{i=1}^{K} R_{i}{ }^{C}
\end{aligned}
$$

Where $P_{i}{ }^{C}$ and $D_{i}{ }^{C}$ demote the charging price and UE's demand respectively when cooperative game model is used.

As regard to the utility function, the quadratic function showed in (7), is also applied. Considering the situation when all the devices set the same charging price, we can derive the demand function as

$$
D_{i}^{C}\left(P_{i}^{C}\right)=\frac{\left(1-P_{i}^{C}\right)}{Q_{i}[\rho(K-1)+1]}
$$

Using the similar solution described in 3.1.3, the optimal charging price $P_{i}^{C^{*}}$ of device $i$ can be obtained as

$$
\frac{\partial R_{i}{ }^{C}(\boldsymbol{P})}{\partial P_{i}^{C}}=0 \Rightarrow P_{i}^{C^{*}}=\frac{1}{2}\left(1+c_{i}\right)
$$

By solving equation (16), the best response price (BRP) $P_{i}^{C *}$ of the each D2D relaying device under cooperative game model can be obtained similarly. 


\section{Numerical Results}

Consider an OFDMA cellular system. It is assumed that cell radius $\mathrm{R}=500 \mathrm{~m}$, UE's transmission range $R_{0}=50 \mathrm{~m}$. The position of each UE is generated randomly within the cell. Suppose that after D2D relaying mode is selected, at least two different D2D devices can provide D2D relaying services for each UE, so it is obtained that $K \geq 2$. We denote these two competing device as device A and device $\mathrm{B}$, respectively. Other parameters used in simulation are listed as: $c_{i}=0.1, r_{i}=0.5,0.3 \leq \rho \leq 0.7,0.4 \leq Q_{i} \leq 0.6$.

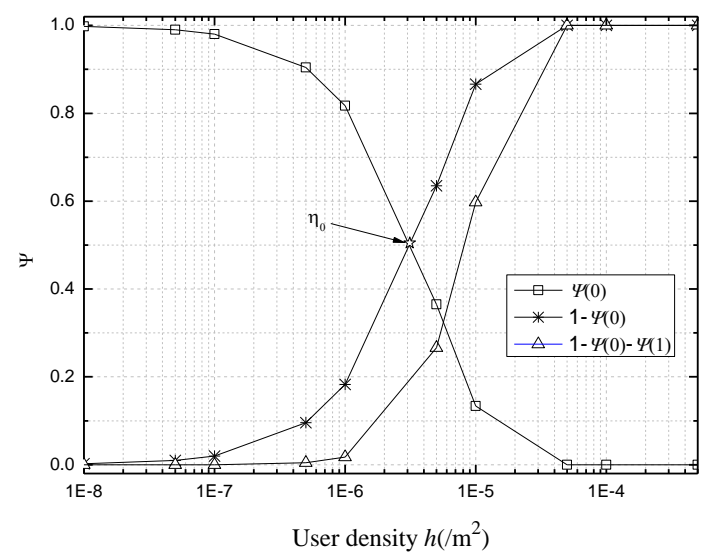

Figure 3. $\psi$ Versus User Density

Figure 3 shows the probability that different number of D2D devices can be found within a UE's effective D2D communication area. This is taken as a function of user density, where $\psi(0)$ denotes the possibility that a certain UE finds no D2D device in its EDCA. The user density $\eta$ in a cell ranges from $10^{-8} / \mathrm{m}^{2}$ (corresponding to $0 \mathrm{D} 2 \mathrm{D}$ device) to $5 \times 10^{-4} / \mathrm{m}^{2}$ (corresponding to $392 \mathrm{D} 2 \mathrm{D}$ devices). According to the expression (4), we can obtain $\eta_{0}$ is equal to $2.1856 \times 10^{-6} / \mathrm{m}^{2}$. From Figure3, it is observed that when user density is about $2.3 \times 10^{-6} / \mathrm{m}^{2}$, then the probability of finding at least one D2D device in a UE's s EDCA is close to 0.5 . This is in accordance with the analysis value. Consequently, only under this situation, should the UE consider to switch into D2D communication mode. When the user density is approximately $3 \times 10^{-5} / \mathrm{m}^{2}$, a UE can definitely find another D2D device in its EDCA.

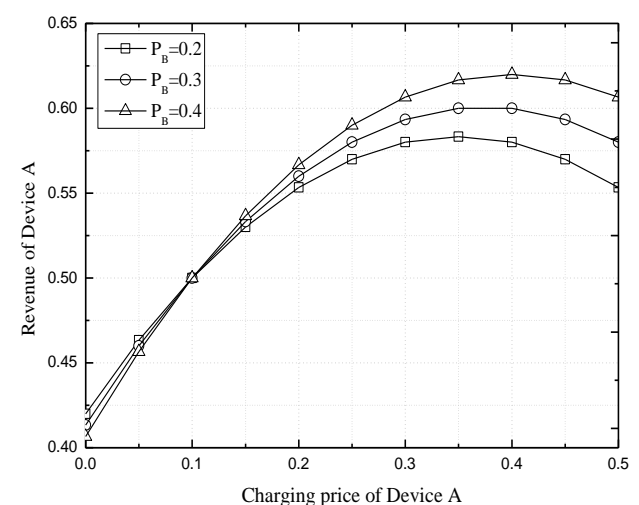

(a)

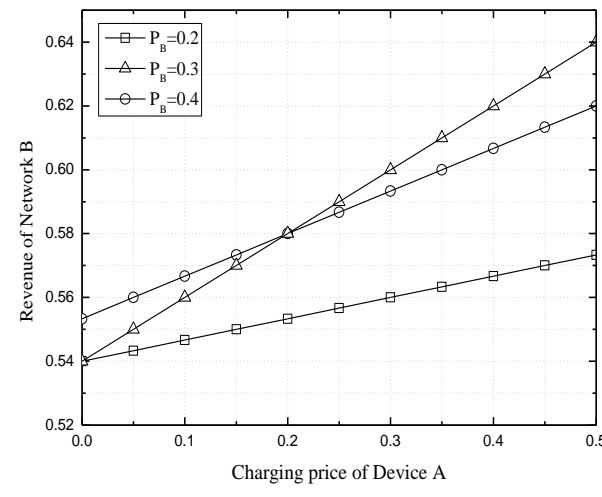

(b)

Figure 4. Revenue of Different Devices 
Figure 4 shows the revenue of different devices when they offering different charging prices. As can be observed in Figure 4(a), given three particular service price of device B, with the varying charging price of device $\mathrm{A}$, the corresponding revenue of device $\mathrm{A}$ also varies. With the same price charged by device $\mathrm{A}$, when the competing device $\mathrm{B}$ offering a higher price (e.g., $\mathrm{P}_{\mathrm{B}}=0.4$ ), more revenue can be obtained by device $\mathrm{A}$. This phenomenon can be explained as more UEs might turn to device A for D2D relaying in order to avoid the high price charged by device B. Moreover, it can be seen that the revenue of device A first increases with the increase of charging price; then after a certain price, the revenue begins to decrease. This certain price is exact what we defined as BCP of device A when the price policy of device $\mathrm{B}$ is settled.

In Figure 4(b), the relationship between the revenue of device B and the charging of device $\mathrm{A}$ is plotted. It is shown that for a certain price settled by device $\mathrm{B}$, the revenue of device $\mathrm{B}$ increases with the increased charging price of device $\mathrm{A}$. This phenomenon can be explained as when the price charged by device A gradually increases, more UE will select device $\mathrm{B}$ as the D2D relaying device for its cheaper price, which leads to the increased revenue of device B. Furthermore, as can be observed that when the charging price of device A is relatively cheap (below 0.2 in the Figure 4(b)), the best price policy of device $\mathrm{B}$ is to adopt a higher price (e.g., $\left.\mathrm{P}_{\mathrm{b}}=0.4\right)$. On the other hand, when device $\mathrm{A}$ adopt a higher price policy, the smart choice of device $\mathrm{B}$ is chosen a cheaper charging price (e.g., $\left.\mathrm{P}_{\mathrm{b}}=0.3\right)$.

From Figure4, it can be inferred that in order to maximize each device's profits, a dynamic price policy should be adopted by both devices. That is, each device should dynamically adjust its anticipatory charging price so as to achieve maximal profit. Thus, the Nash equilibrium price benefited for both participants in the game can be found.

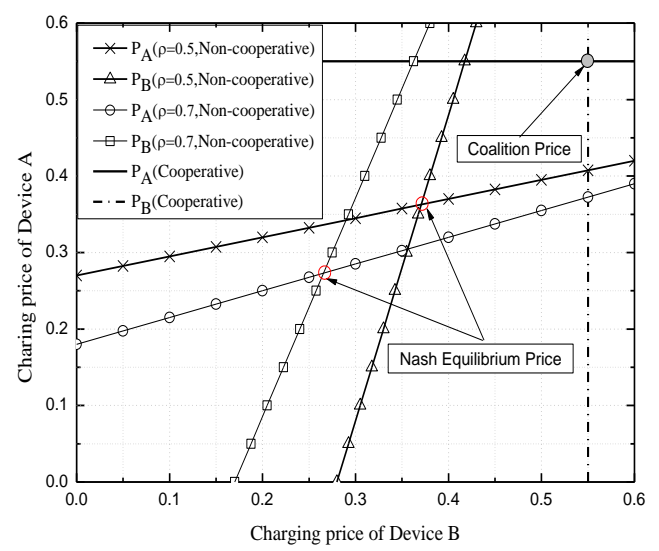

Figure 5. Nash Equilibrium Price and Coalition Price

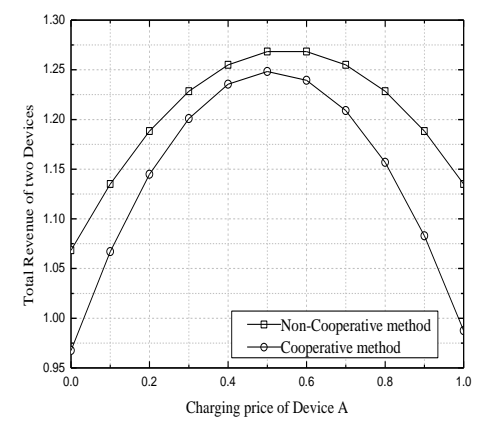

Figure 6. Total Revenue of Two Devices 
Figure 5 shows the comparison of Nash equilibrium prices under non-cooperative game, as well as coalition price of cooperative game with the same network condition. As can be observed, for non-cooperative game, by first plotting the optimal charging price $P_{i}{ }^{*}$ described in function (12), the BCP of two devices can be obtained. The Nash equilibrium price is described as the intersection of the BCP of two devices. Since the BCP of two devices are linear functions shown in equation (12), the Nash equilibrium price under any network scenario exists and is unique. Moreover, when the competition between the two devices is intensified (lager $\rho$ adopted), it can be seen from the figure that the Nash equilibrium price also decrease. This phenomenon could be explained as: when the competition is becoming fierce among D2D device, in order to attract more UE to select the device as D2D relaying service provider, the best strategy is to decrease charging price so as to be more competitive. As for cooperative game, the coalition price is obtained by adopted equation (16). It can be obvious seen that the coalition price is higher than the Nash equilibrium price. This situation is owing to the fact that under cooperative game, since there is no competition among D2D devices, the maximal charging price is obtained under monopolization.

Figure6 shows the total revenue of two devices under different game model. It can be observed that with the adoption of coalition price, the total revenue is maximized when D2D devices cooperate with each other. While for non-cooperative method, due to intense competition among devices, each D2D device only aims at maximize its own profits, which leads to reduced total revenue compared with cooperative model. Moreover, with the increase of charging price of Device A, the difference of total revenue under two different game models first become smaller, and then gradually become larger. This phenomenon can be interpreted as: for non-cooperative method, with the increase of charging price of device A, Nash equilibrium price is gradually achieved which leads to the maximal total revenue. While for cooperative method, the coalition price is also arrived with the increase of charging price of device A, hence leads to the maximized total revenue.

\section{Conclusion}

In this paper, the mode selection issues as well as pricing mechanism in deviceto-device relaying communications is discussed. We first explore the problem of "when D2D relaying mode should be selected?" By considering the user density within the cell, we point out the exact condition under which should a user change into D2D communication model. Furthermore, based on non-cooperative game theory as well as cooperative game theory, we propose the optimal pricing mechanism for D2D communication peers to answer the question of "How should the D2D relaying behavior be encouraged?" The proposed mechanism is based on the user's payoff model, which both considers user's demands and D2D devices' constraint ability. Simulation results demonstrate that under game theoretical model, the proposed scheme can achieve the maximal utility for D2D devices when the $\mathrm{BCP}$ price is adopted, hence better user experience can be obtained.

\section{Acknowledgements}

This work is supported in part by the National 863 High Tech R\&D Program of China (project number: 2014AA01A703, National Science and Technology Major Project of the Ministry of Science and Technology of China(project number: 2014ZX03001025-006), Science and Technology Research and Development Program of Shaanxi Province (project number: 2014K09-14). 


\title{
References
}

[1] D. Astely, E. Dahlman, A. Furuskar, Y. Jading, M. Lindstrom and S. Parkvall, "LTE: the evolution of mobile broadband", IEEE Communications Magazine, vol. 47, no.4, (2009), pp. 44-51.

[2] I. F. Akyildiz, D. M.Gutierrez-Estevez, R. Balakrishnan and E. Chavarria-Reyes, "LTE-Advanced and the evolution to Beyond 4G (B4G) systems", Physical Communication, vol. 10, (2014), pp. 31-61.

[3] G Fodor, E. Dahlman, G. Mildh, S. Parkvall, N. Reider, G. Miklos, and Z. Turanyi, "Design Aspects of Network Assisted Device-to-Device Communications", IEEE Communications Magazine, vol. 50, no. 3, (2012), pp. 170-77.

[4] D. Q. Feng, L. Lu, Y. Y. Wu, G. Li, S. Q. Li, and G. Feng, "Device-to-device communications Underlaying Cellular Networks", IEEE Transaction on Communications, vol. 61, no. 8, (2013), pp. 3541-3551.

[5] K. Doppler, M. Rinne, C. Wijting, C. Ribeiro and K. Hugl, "Device-to-device communication as an underlay to LTE-Advanced networks", IEEE Communications Magazine, vol. 47, no. 12, (2009), pp. $42-49$.

[6] M. N. Tehrani, M. Uysal and H. Yanikomeroglu, "Device-to-Device Communication in 5G Cellular Networks: Challenges, Solutions, and Future Directions", IEEE Communications Magazine, vol. 52, no. 5, (2014), pp. 49-55.

[7] S. Hakola, C. Tao, J. Lehtomaki and T. Koskela, "Device-to-Device (D2D) Communication in Cellular Network - Performance Analysis of Optimum and Practical Communication Mode Selection", Proceedings of the 2010 IEEE Wireless Communications and Networking Conference, Sydney, NSW, (2010), pp. 1-6.

[8] G. Zhang, K. Yang, P. Liu and J. Wei, "Power Allocation for Full-Duplex Relaying Based D2D Communication Underlying Cellular Networks", IEEE Transaction on Vehicular Technology, no. 99, (2014), pp. 1-7.

[9] K. D. Lee and V. C. M. Leung, "Evaluations of Achievable Rate and Power Consumption in Cooperative Cellular Networks with Two Classes of Nodes", IEEE Transactions on Vehicular Technology, vol. 57, no. 2, (2008), pp. 1166-1175.

[10] Q. B. Chen, W. G. Zhou, R. Chai and L. Tang, "Game-theoretic approach for pricing strategy and network selection in heterogeneous wireless networks", IET Communications, vol. 5, no. 5, (2011), pp. 676-682.

[11] S. Sengupta, M. Chatterjee and K. A. Kwiat, "A game theoretic framework for power control in wireless sensor networks", IEEE Transactions on Computers, vol. 59, no. 2, (2010), pp. 231-242.

[12] F. Jiang, H. Hui and P. Zhang, "A user cooperation stimulating strategy based on cooperative game theory in cooperative relay networks", EURASIP Journal on Wireless Communications and Networking, vol. 2009,(2009), pp. 1-11.

\begin{abstract}
Authors
Fan Jiang: Fan Jiang is an Associated Professor with the school of communication and information Engineering, Xian University of Posts and Telecommunications, Shaanxi, China. She received her BS and MS degrees from Xidian University, China, in 2004 and 2007. She received her $\mathrm{PhD}$ degree in 2010 from Beijing University of Posts and Telecommunications, China, in June. Since 2010, she has been working at Xian University of Posts and Telecommunications. She has authored number of national and international papers and articles in reputed journals and conferences. Her major research interest lies in wireless communication systems, including MAC protocols design for LTE and LTE-Advanced, D2D communications in 5G system, heterogeneous networks, cooperative communications, relay networks, random access algorithms.

Benchao Wang: Benchao Wang is an engineer at Shaanxi radio monitoring station, State Radio Regulatory of China. He received his MS degrees in communication and information system from Xidian University, China, in 2007. From 2007 to 2010, he serves as a research engineer in Haige Communications Group Incorporated Company. From 2010 to 2013, he joined Huawei Technologies as a research staff. His current research interests lies in the fields of multi-hop transmissions, cognitive radio, and their applications in future $5 \mathrm{G}$ mobile communication system.
\end{abstract}


Changyin Sun: Changyin Sun is an Associated Professor with the school of communication and information Engineering, Xian University of Posts and Telecommunications, Shaanxi, China. He received the PhD degree from the National Key Laboratory of Radar Signal Processing (RSP), Xidian University, P.R. China, in 2000. From 2001 to 2010, he was a senior engineer at ZTE Corporation, where he worked on the R\&D and pre-research of the evolved mobile communication system. Since 2010, he has held the position of Associated Professor with the Department of Telecommunication Engineering, Xian University of Posts and Telecommunications, P.R. China. His research area includes interference management and radio resource management in the evolved mobile communication system. 